\title{
Commentary: The Flavonoid Baicalein Rescues Synaptic Plasticity and Memory Deficits in a Mouse Model of Alzheimer's Disease
}

\author{
Salvatore Chirumbolo ${ }^{1 *}$ and Geir Bjørklund ${ }^{2}$ \\ ${ }^{1}$ Department of Neurological and Movement Science, University of Verona, Verona, Italy, ${ }^{2}$ Council for Nutritional and \\ Environmental Medicine, Mo i Rana, Norway
}

Keywords: Alzheimer disease, synaptic loss, GSK-3ß, baicalein, flavonoids, astrocyte, glutamate receptors

\section{A commentary on}

The Flavonoid Baicalein Rescues Synaptic Plasticity and Memory Deficits in a Mouse Model of Alzheimer's Disease

by Gu XH, Xu LJ, Liu ZQ, Wei B, Yang YJ, Xu GG, et al. Behav Brain Res (2016) 311:309-21. doi: $10.1016 / j . b b r .2016 .05 .052$

OPEN ACCESS

Edited by: Irving E. Vega,

Michigan State University, USA

Reviewed by:

Hector De Jesus-Cortes,

Massachusetts Institute of

Technology, USA

Manoj Kumar Jaiswal,

Columbia University, USA

*Correspondence:

Salvatore Chirumbolo salvatore.chirumbolo@univr.it

Specialty section: This article was submitted to Neurodegeneration, a section of the journal

Frontiers in Neurology

Received: 31 May 2016 Accepted: 17 August 2016 Published: 29 August 2016

Citation: Chirumbolo S and Bjørklund G (2016) Commentary: The Flavonoid

Baicalein Rescues Synaptic Plasticity and Memory Deficits in a Mouse Model of Alzheimer's Disease. Front. Neurol. 7:141. doi: 10.3389/fneur.2016.00141
A recent article by $\mathrm{Gu}$ et al. reported that the long-term oral administration of baicalein in mouse, inhibited the activity of lipooxygenase 12/15 LXO and glycogen synthase kinase $3 \beta$ (GSK-3 $\beta$ ) in hippocampal slices, also reducing the activity of $\beta$-secretase enzyme (BACE1) and the concentration of total beta-amyloid proteins $(\mathrm{A} \beta)$, leading to the in vivo restoration of spine number, synaptic plasticity, and memory deficits (1).

Baicalein (5,6,7-trihydroxy-2-phenyl-chromen-4-one) is present in the plant genus Scutellaria, e.g., Scutellaria baicalensis, the flavone recently went in the spotlight for its ability in preventing Alzheimer disease $(\mathrm{AD})$ and other neurodegenerative disorders (2-4). According to the authors, baicalein is able to prevent the impairment in the hippocampal long-term potentiation (LTP) induced by $\beta$-amyloids $(\mathrm{A} \beta)$ and improve cognitive deficit associated with $\mathrm{AD}(1)$. Recently, also metformin exhibited positive effects on $\mathrm{AD}$ quite comparable to baicalein, i.e., the inhibition of the activity of A $\beta$ on LTP in a high fat diet (HFD) rat model (5). As evidence about the anti-obesity and anti-diabetes activity of baicalein was reported elsewhere, the activity of metformin may give insights on the role exerted by baicalein in AD. Despite the evidence reported by Asadbegi et al. some controversial result yet exists, as in type 2 diabetes models metformin increases the expression of the $\beta$-amyloid precursor protein (APP), through the activation of NF- $\mathrm{kB}$, causing accumulation of $\mathrm{A} \beta$ in rat brain $(5,6)$. Whether metformin has either a beneficial or a noxious effect depending on the cellular system considered or if it acts through a typical bimodal mechanism, a hallmark shared by several bioflavonoids, is yet far to be fully elucidated. As like as flavonoids, even metformin, which is a well known anti-diabetic drug, possesses anti-oxidant and anti-inflammatory properties (7). This recent evidence suggested us a possible mechanism for baicalein action in AD prevention, as also for the flavonoid baicalein, a bimodal activity might be addressed, at least as concern its action on oxidative stress and metabolism. The main advantage respect to metformin is that baicalein is a plant-derived natural compound. As many bioflavonoids, baicalein inhibits phosphorylation of many fundamental signaling protein kinases, leading to its typical anti-inflammatory activity, e.g., it inhibits the ERK/MAPK signaling cascade, acting on the phosphorylation of MEK-1 by Raf-1 and inducing dampening of NF- $\mathrm{\kappa B}(8)$. This anti-phoshorylation property was reported also by Gu et al. as baicalein should exert its action by preventing the phosphorylation of tau protein in APP/PS1 mice (1). Still, a metabolic, anti-oxidant activity from baicalein, besides its targeting glutamatergic neurotransmission, may explain some evidence reported (1). 
The authors showed that $\mathrm{A} \beta$ binding to synapse-related sites mediates the reduction of synaptic loss and also modulates the damage of the glutamatergic synaptic transmission, while baicalein can prevent this damage (1). Furthermore, the paper by $\mathrm{Gu}$ et al. would suggest whether the mechanism by which baicalein acts on $\mathrm{AD}$ may resemble some other neuro-protective compound, in order to focus onto commonly shared targets. Curiously, as like as metformin, also baicalein succeeded in rescuing hippocampal LTP impaired by the action of $A \beta$ and the authors suggested a mechanism working through the PI3K signaling pathway $(1,5)$. Further bioflavonoids seem to have a comparable effect on the synaptic failure induced by $\mathrm{A} \beta$, e.g., the glycosylated-flavonol troxerutin, is able also to reduce impairments in cognitive function caused by metabolic syndrome or $\operatorname{HFD}(9,10)$ and the flavone nobiletin, from Citrus fruit, rescued cognitive function and memory impairment in animal fear conditioning tests by reverting the inhibition caused by $A \beta$ on the membrane trafficking of glutamate receptors, which is required for LTP (11). And even nobiletin has an impact on obesity and metabolic syndrome (12). The question is if there is a fundamental connection between metabolic syndrome and neurodegenerative disorders in the reported action of baicalein.

A possible mechanism linking these effects and shared by metformin besides flavonoids might involve their indirect action on the impaired insulin signaling and glucose metabolism, which causes $\mathrm{AD}$ in brain (13). For example, the paper by $\mathrm{Gu}$ et al. showed that baicalein inhibits the activity of GSK-3 $\beta$. Brain derived insulin is expressed by the activity of the transcription factor neurogenic differentiation 1 (NeuroD1) and inhibitors of GSK-3 $\beta$ increase insulin gene (Ins2) and NeuroD1 in the mouse brain, through a Wnt/ $\beta$-catenin pathway (14). Gu et al. also reported that baicalein increased the $N$-methyl-D-aspartate glutamate receptor (NMDAR)-mediated LTP in rat hippocampus and it is well known that glutamate, which is spontaneously released by astrocytes, is involved in the setting of a LTP threshold (15). Therefore, it is possible that an activity of baicalein described by $\mathrm{Gu}$ et al. may target the insulin signaling by inhibiting the GSK-3 $\beta$ pathway.

\section{REFERENCES}

1. Gu XH, Xu LJ, Liu ZQ, Wei B, Yang YJ, Xu GG, et al. The flavonoid baicalein rescues synaptic plasticity and memory deficits in a mouse model of Alzheimer's disease. Behav Brain Res (2016) 311:309-21. doi:10.1016/j.bbr.2016. 05.052

2. Wei D, Tang J, Bai W, Wang Y, Zhang Z. Ameliorative effects of baicalein on an amyloid- $\beta$ induced Alzheimer's disease rat model: a proteomics study. Curr Alzheimer Res (2014) 11(9):869-81. doi:10.2174/1567205011666141001113619

3. Zhang SQ, Obregon D, Ehrhart J, Deng J, Tian J, Hou H, et al. Baicalein reduces $\beta$-amyloid and promotes nonamyloidogenic amyloid precursor protein processing in an Alzheimer's disease transgenic mouse model. J Neurosci Res (2013) 91(9):1239-46. doi:10.1002/jnr.23244

4. Xue X, Liu H, Qi L, Li X, Guo C, Gong D, et al. Baicalein ameliorated the upregulation of striatal glutamatergic transmission in the mice model of Parkinson's disease. Brain Res Bull (2014) 103:54-9. doi:10.1016/j.brainresbull. 2014.02.004

5. Asadbegi M, Yaghmaei P, Salehi I, Ebrahim-Habibi A, Komaki A. Neuroprotective effects of metformin against $A \beta$-mediated inhibition
The most acknowledged activity of plants bioflavonoids is their targeting oxidative stress, gene transcription factors involved in the cellular response to oxidative stress and mechanisms involved in the scavenging of oxidative stressors. Actually, it is not surprising that for the interesting evidence reported by $\mathrm{Gu}$ et al. the anti-oxidant property of baicalein might even play a major role. For example, the activity of membrane PI3K in astrocytes, through the up-regulation of the signaling mechanism, involving the activation of phospholipase $\mathrm{C}$ and protein kinase C-delta (PKC- $\delta$ ), causes a weak but persistent stimulation of the $\mathrm{N}$-methyl-D-aspartate receptors (NMDARs) from astrocytes and this mechanism is coupled with the phosphorylation at $\operatorname{Thr}(395)$, Ser(433), and Thr(439) by PKC- $\delta$ of the nuclear factor-(erythroidderived 2)-like 2 (Nrf2), then linking glutamate transmission with anti-oxidant activity and neuron survival. Translocation to the nucleus of Nrf2 enhances the NMDAR stimulation (16). Baicalein may target this mechanism, in the system described by $\mathrm{Gu}$ et al. This should suggest that a possible effect of baicalein may be exerted also on the glia, besides neurons, particularly during the inflammatory response (17). In this circumstance, further research should address the widespread opinion that plant polyphenols act on more than one level, fundamentally by targeting signaling system that are involved in cell survival and stress response. This would involve the existence of complex machinery encompassing both homeostasis of the energetic balance and cell response to stressors. In this sense, the bio-molecular mechanism by which baicalein caused the evidence reported by Gu et al. may mainly depend on its anti-oxidant property, as $\beta$-secretase 1 (BACE1) activity, which increases the level of APP, is promoted by pro-oxidant factors (18). This potential should shed a light also on the neuroprotective role of those natural compounds, which are recognized as anti-diabetic and obesity-preventive molecules.

\section{AUTHOR CONTRIBUTIONS}

SC conceived the paper, SC and GB read the literature, GB contribute in the editing of the document, $\mathrm{SC}$ wrote with GB the manuscript, SC and GB revised the first draft, SC submitted the manuscript.

of long-term potentiation in rats fed a high-fat diet. Brain Res Bull (2016) 121:178-85. doi:10.1016/j.brainresbull.2016.02.005

6. Picone P, Nuzzo D, Caruana L, Messina E, Barera A, Vasto S, et al. Metformin increases APP expression and processing via oxidative stress, mitochondrial dysfunction and NF- $\mathrm{\kappa B}$ activation: use of insulin to attenuate metformin's effect. Biochim Biophys Acta (2015) 1853(5):1046-59. doi:10.1016/j.bbamcr. 2015.01.017

7. Porceddu PF, Ishola IO, Contu L, Morelli M. Metformin prevented dopaminergic neurotoxicity induced by 3,4-methylenedioxymethamphetamine administration. Neurotox Res (2016) 30(1):101-9. doi:10.1007/ s12640-016-9633-5

8. Nakahata N, Tsuchiya C, Nakatani K, Ohizumi Y, Ohkubo S. Baicalein inhibits Raf-1-mediated phosphorylation of MEK-1 in C6 rat glioma cells. Eur J Pharmacol (2003) 461(1):1-7. doi:10.1016/S0014-2999(02)02950-3

9. Babri S, Mohaddes G, Feizi I, Mohammadnia A, Niapour A, Alihemmati A, et al. Effect of troxerutin on synaptic plasticity of hippocampal dentate gyrus neurons in a $\beta$-amyloid model of Alzheimer's disease: an electrophysiological study. Eur J Pharmacol (2014) 732:19-25. doi:10.1016/j.ejphar. 2014.03.018 
10. Lu J, Wu DM, Zheng ZH, Zheng YL, Hu B, Zhang ZF. Troxerutin protects against high cholesterol-induced cognitive deficits in mice. Brain (2011) 134(Pt 3):783-97. doi:10.1093/brain/awq376

11. Yamakuni T, Nakajima A, Ohizumi Y. [Preventive action of nobiletin, a constituent of AURANTII NOBILIS PERICARPIUM with anti-dementia activity, against amyloid-beta peptide-induced neurotoxicity expression and memory impairment]. Yakugaku Zasshi (2010) 130(4):517-20. doi:10.1248/ yakushi.130.517

12. Lee YS, Cha BY, Choi SS, Choi BK, Yonezawa T, Teruya T, et al. Nobiletin improves obesity and insulin resistance in high-fat diet-induced obese mice. J Nutr Biochem (2013) 24(1):156-62. doi:10.1016/j.jnutbio.2012.03.014

13. Chen Y, Zhang J, Zhang B, Gong CX. Targeting insulin signaling for the treatment of Alzheimer's Disease. Curr Top Med Chem (2016) 16(5):485-92. doi:10.2174/1568026615666150813142423

14. Lee J, Kim K, Yu SW, Kim EK. Wnt3a upregulates brain-derived insulin by increasing NeuroD1 via Wnt/ $\beta$-catenin signaling in the hypothalamus. Mol Brain (2016) 9:24. doi:10.1186/s13041-016-0207-5

15. Bonansco C, Couve A, Perea G, Ferradas CÁ, Roncagliolo M, Fuenzalida M. Glutamate released spontaneously from astrocytes sets the threshold for synaptic plasticity. Eur JNeurosci (2011) 33(8):1483-92. doi:10.1111/ j.1460-9568.2011.07631.x
16. Jimenez-Blasco D, Santofimia-Castaño P, Gonzalez A, Almeida A, Bolaños JP. Astrocyte NMDA receptors' activity sustains neuronal survival through a Cdk5-Nrf2 pathway. Cell Death Differ (2015) 22(11):1877-89. doi:10.1038/ cdd.2015.49

17. Hong S, Beja-Glasser VF, Nfonoyim BM, Frouin A, Li S, Ramakrishnan S, et al. Complement and microglia mediate early synapse loss in Alzheimer mouse models. Science (2016) 352(6286):712-6. doi:10.1126/science.aad8373

18. Choudhry F, Howlett DR, Richardson JC, Francis PT, Williams RJ. Prooxidant diet enhances $\beta / \gamma$ secretase-mediated APP processing in APP/PS1 transgenic mice. Neurobiol Aging (2012) 33(5):960-8.

Conflict of Interest Statement: The authors declare that the research was conducted in the absence of any commercial or financial relationships that could be construed as a potential conflict of interest.

Copyright (C) 2016 Chirumbolo and Bjørklund. This is an open-access article distributed under the terms of the Creative Commons Attribution License (CC BY). The use, distribution or reproduction in other forums is permitted, provided the original author(s) or licensor are credited and that the original publication in this journal is cited, in accordance with accepted academic practice. No use, distribution or reproduction is permitted which does not comply with these terms. 